\title{
Positive Steady States of a Strongly Coupled Predator-Prey System with Holling- $(n+1)$ Functional Response
}

\author{
Xiao-zhou Feng ${ }^{1,2}$ and Zhi-guo Wang ${ }^{2}$ \\ ${ }^{1}$ College of Science, Xi'an Technological University, Xi'an 710032, China \\ ${ }^{2}$ Institute of Mathematics, Shaanxi Normal University, Xian 710062, China \\ Correspondence should be addressed to Xiao-zhou Feng; flxzfxz8@163.com
}

Received 25 December 2012; Accepted 2 March 2013

Academic Editor: Junjie Wei

Copyright (c) 2013 X.-z. Feng and Z.-g. Wang. This is an open access article distributed under the Creative Commons Attribution License, which permits unrestricted use, distribution, and reproduction in any medium, provided the original work is properly cited.

This paper discusses a predator-prey system with Holling- $(n+1)$ functional response and the fractional type nonlinear diffusion term in a bounded domain under homogeneous Neumann boundary condition. The existence and nonexistence results concerning nonconstant positive steady states of the system were obtained. In particular, we prove that the positive constant solution $(\widetilde{\mathcal{u}}, \widetilde{v})$ is asymptotically stable when the parameter $k$ satisfies some conditions.

\section{Introduction}

In this paper, we are interested in the positive steady states of the strongly coupled predator-prey system with Holling$(n+1)$ functional response. The specific system is as follows:

$$
\begin{gathered}
-d_{1} \Delta u=u\left(1-\frac{u}{k}\right)-\frac{u^{n} v}{a+u^{n}} \quad \text { in } \Omega, \\
-d_{2} \Delta\left(v+\frac{d_{3} v}{1+u}\right)=-b v+\frac{\sigma u^{n} v}{a+u^{n}} \quad \text { in } \Omega, \\
\frac{\partial u}{\partial v}=\frac{\partial v}{\partial v}=0 \quad \text { on } \partial \Omega,
\end{gathered}
$$

where $1 \leq n<+\infty$; $\Omega$ is a bounded domain in $R^{N}$ with smooth boundary $\partial \Omega ; \partial / \partial \nu$ is the outward directional derivative normal to $\partial \Omega ; u$ and $v$ stand for the densities of the prey and predator; the given coefficients $d_{i}(i=1,2)$, $a, b, k$, and $\sigma$ are positive constants. The term $u^{n} /\left(a+u^{n}\right)$ is named Holling- $(n+1)$ functional response $[1,2]$. In the second equation, the fractional type nonlinear diffusion term $\Delta d_{2}\left(d_{3} v /(1+u)\right)$ models a situation in which the population pressure of the predator species weakens in high-density areas of the prey species. For more precise details, we can refer to $[3,4]$. Paper [3] discusses a strongly coupled predatorprey system with nonmonotonic functional response, the existence and nonexistence results concerning nonconstant positive steady states of the system were proved by degree theory. Paper [4] considers the positive steady states for a prey-predator model with some nonlinear diffusion terms, and the sufficient conditions for the existence of positive steady state solutions were obtained by bifurcation theory.

In recent years, there has been considerable interest in the dynamics of strongly coupled reaction-diffusion systems with cross-diffusion. We point out that most efforts have concentrated on the Lotka-Volterra competition system which was proposed first by Shigesada et al. [5]. Since their pioneering work, many authors have studied population models with cross-diffusion terms from various mathematical viewpoints, for example, the global existence of time-depending solutions [6-11], the stability analysis for steady states [12-14], and the steady state problems [15-21]. In this paper, we mainly consider the existence of solutions of (1). The research method refers to $[3,22,23]$.

For convenience of the research, we write (1) as the following form:

$$
-\Delta \Phi(U)=G(U), \quad U=\left[\begin{array}{l}
u \\
v
\end{array}\right]
$$


where

$$
\begin{gathered}
\Phi(U)=\left[\begin{array}{l}
\phi_{1}(U) \\
\phi_{2}(U)
\end{array}\right]=\left[\begin{array}{c}
d_{1} u \\
d_{2} v\left(1+\frac{d_{3}}{1+u}\right)
\end{array}\right], \\
G(U)=\left[\begin{array}{c}
g(u)-v p(u) \\
-b v+\sigma v p(u)
\end{array}\right], \\
g(u)=u\left(1-\frac{u}{k}\right), \quad p(u)=\frac{u^{n}}{a+u^{n}}, \quad n \in N^{+} .
\end{gathered}
$$

The main work of this paper is to study the effects of the fractional type nonlinear diffusion pressures on the existence of nonconstant positive steady states of (1). Here, a positive solution means a smooth solution $(u, v)$ with both $u$ and $v$ being positive. We will demonstrate that the cross-diffusion pressure $d_{3}$ may help forming more patterns. Obviously, for system (1), one notes that when $\sigma \leq b$, there holds $\sup _{s \geq 0}\{-b+$ $\left.\sigma s^{n} /\left(a+s^{n}\right)\right\} \leq 0$, so that the only nonnegative solutions to (1) are $U=(0,0)$ and $U=(k, 0)$. Consequently, (1) does not have any positive solution. On the other hand, when $\sigma>b$, the unique positive constant solution to (1) is $(\widetilde{u}, \widetilde{v})$; that is,

$$
\begin{gathered}
\widetilde{u}=\sqrt[n]{\frac{a b}{\sigma-b}}, \\
\widetilde{v}=\frac{(1-\widetilde{u} / k)\left(a+\widetilde{u}^{n}\right)}{\widetilde{u}^{n-1}}=\frac{(k-\widetilde{u}) \sigma \widetilde{u}}{k b}, \quad \text { if } k>\widetilde{u} .
\end{gathered}
$$

The organization of our paper is as follows. In Section 2, we establish a priori upper and lower bounds for positive solutions of (1). In Section 3, we use a degree theory to develop a general result to enable one to conclude the existence or nonexistence of nonconstant steady-state solutions or patterns as the index of positive constant steady states changes. In Section 4, we establish the existence of nonconstant positive solutions to (1) for a large range of diffusion and cross-diffusion coefficients. Meanwhile, we prove that the positive constant solution $(\widetilde{u}, \widetilde{v})$ is asymptotically stable for different ranges of parameters.

\section{Upper and Lower Bounds for Positive Solutions}

The main purpose of this section is to give a priori upper and lower positive bounds for positive solutions of (1). Firstly, we cite two known results.

Harnack Inequality (see [24]). Let $c \in C(\bar{\Omega})$ and $\omega \in$ $C^{2}(\Omega) \cap C^{1}(\bar{\Omega})$ be a positive classical solution to $\Delta \omega(x)+$ $c(x) \omega(x)=0$ in $\Omega$ subject to the homogeneous Neumann boundary condition. Then, there exists a positive constant $C=C\left(\Omega,\|c\|_{\infty}\right)$ such that $\max _{\bar{\Omega}} \omega \leq C \min _{\bar{\Omega}} \omega$.

Maximum Principle (see [25]). Suppose that $g \in C\left(\bar{\Omega} \times R^{1}\right)$. If $w \in C^{2}(\Omega) \cap C^{1}(\bar{\Omega})$ satisfies

$$
\begin{gathered}
\Delta w(x)+g(x, w(x)) \geq 0 \quad \text { in } \Omega, \\
\frac{\partial w}{\partial \nu} \leq 0 \quad \text { on } \partial \Omega,
\end{gathered}
$$

and $w\left(x_{0}\right)=\max _{\bar{\Omega}} w$, then $g\left(x_{0}, w\left(x_{0}\right)\right) \geq 0$.

Theorem 1. Let $P=(\sigma, a, k, b) \in(0, \infty)^{4}$ and $d, \widehat{d}>0$ be fixed constants. Assume that $d_{1}, d_{2} \geq d$ and $0 \leq d_{3} \leq \widehat{d}$ there exists a positive constant $C=C(P, d, \widehat{d}, \Omega)$, such that any positive solution $(u, v)$ of $(1)$ satisfies

$$
\max _{\bar{\Omega}} u(x)+\max _{\bar{\Omega}} v(x)+\frac{\max _{\bar{\Omega}} u(x)}{\min _{\bar{\Omega}} u(x)}+\frac{\max _{\bar{\Omega}} v(x)}{\min _{\bar{\Omega}} v(x)} \leq C .
$$

Proof. Let $\phi_{1}(U)$ and $\phi_{2}(U)$ be defined as in (3), and denote that

$$
\begin{gathered}
g_{1}(U)=u\left(1-\frac{u}{k}-\frac{u^{n-1} v}{a+u^{n}}\right), \\
g_{2}(U)=v\left(\frac{\sigma u^{n}}{a+u^{n}}-b\right), \\
G(U)=\left(g_{1}(U), g_{2}(U)\right)^{T} .
\end{gathered}
$$

Then, (1) becomes

$$
-\Delta \Phi(U)=G(U) \quad \text { in } \Omega, \quad \frac{\partial U}{\partial \nu}=0 \quad \text { on } \partial \Omega .
$$

For the first equation of (9), by the Maximum Principle, we have

$$
\max _{\bar{\Omega}} u(x) \leq \frac{1}{d_{1}} \max _{\bar{\Omega}} \phi_{1}(U)<k .
$$

The function $\phi_{2}(U)$ satisfies

$$
\begin{gathered}
\Delta \phi_{2}(U)+c(x) \phi_{2}(U)=0 \quad \text { in } \Omega, \\
\frac{\partial \phi_{2}(U)}{\partial v}=0 \quad \text { on } \partial \Omega,
\end{gathered}
$$

where $c(x)=g_{2}(U) / \phi_{2}(U) \in C(\bar{\Omega})$. It is easy to verify that the norm

$$
\begin{aligned}
\|c\|_{\infty} & =\left\|\frac{v\left(\sigma u^{n} /\left(a+u^{n}\right)-b\right)}{d_{2}\left(v+d_{3} v /(1+u)\right)}\right\|_{\infty} \leq \frac{\sigma k^{n} /\left(a+k^{n}\right)+b}{d_{2}} \\
& \leq \frac{\sigma k^{n}+b\left(a+k^{n}\right)}{d\left(a+k^{n}\right)}
\end{aligned}
$$


then, $\|c\|_{\infty}$ is bounded by a constant depending only on $P$ and $d\left(d_{2} \geq d\right)$. By the Harnack inequality, we have

$$
\max _{\bar{\Omega}} \phi_{2}(U(x)) \leq C_{1} \min _{\bar{\Omega}} \phi_{2}(U(x)),
$$

where $C_{1}=C_{1}\left(\Omega,\|c\|_{\infty}\right)=C_{1}(P, d, \Omega)$. It follows that

$$
\begin{aligned}
\frac{\max _{\bar{\Omega}} v}{\min _{\bar{\Omega}} v} & \leq \frac{\max _{\bar{\Omega}} \phi_{2}(U(x))}{\min _{\bar{\Omega}} \phi_{2}(U(x))} \times \frac{\max _{\bar{\Omega}}\left\{1+d_{3}(1+u)^{-1}\right\}}{\min _{\bar{\Omega}}\left\{1+d_{3}(1+u)^{-1}\right\}} \\
& \leq C_{1}\left(1+d_{3}\right) \leq C_{1}(1+\hat{d}) .
\end{aligned}
$$

Integrating the equations of (1) over $\Omega$ by parts and making use of the boundary conditions, we have

$$
\sigma \int_{\Omega} u\left(1-\frac{u}{k}\right) d x=b \int_{\Omega} v d x
$$

For any $f \in L^{1}(\Omega)$, we denote that $\bar{f}=(1 /|\Omega|) \int_{\Omega} f(x) d x$. Then, from (15) and (10), we have

$$
\bar{v}=\frac{\sigma}{|\Omega| b} \int_{\Omega} u\left(1-\frac{u}{k}\right) d x<\frac{\sigma}{b} k .
$$

Along with (14), we have

$$
\max _{\bar{\Omega}} v \leq C_{1}(1+\widehat{d}) \frac{\sigma}{b} k .
$$

Similarly, consider the equation of $\phi_{1}$ as follows:

$$
\begin{aligned}
\|c\|_{\infty} & =\frac{1}{d_{1}}\left\|1-\frac{u}{k}-\frac{u^{n-1} v}{a+u^{n}}\right\|_{\infty} \\
& \leq \frac{1}{d_{1}}\left[1+\frac{1}{k}\|u(x)\|_{\infty}+m\|v(x)\|_{\infty}\right],
\end{aligned}
$$

where $m=[(n-1) a]^{(n-1) / n} / n a$. Then, $\|c\|_{\infty}$ is bounded by a constant depending only on $P$ and $d\left(d_{1} \geq d\right)$. By the Harnack Inequality, there exists a positive constant $C_{2}=$ $C_{2}(P, d, \widehat{d}, \Omega)$ such that $\max _{\bar{\Omega}} \phi_{1}(U(x)) \leq C_{2} \min _{\bar{\Omega}} \phi_{1}(U(x))$. Then,

$$
\frac{\max _{\bar{\Omega}} u}{\min _{\bar{\Omega}} u}=\frac{\max _{\bar{\Omega}} \phi_{1}(U(x))}{\min _{\bar{\Omega}} \phi_{1}(U(x))} \leq C_{2} .
$$

Thus, along with (10), (14), and (17), we can complete the proof.

Theorem 2. Assume that $\sigma k^{n} \neq b\left(a+k^{n}\right)$. Let $d, \widehat{d}>0$ be fixed constants. There exists a constant $\underline{C}=\underline{C}(d, \widehat{d}, P, \Omega)>0$, such that any positive solution $(u, v)$ of $(1)$ satisfies

$$
\min _{\bar{\Omega}} u(x)>\underline{C}, \quad \min _{\bar{\Omega}} v(x)>\underline{C},
$$

provided that $d_{1}, d_{2} \geq d$ and $d_{3} \leq \widehat{d}$.
Proof. Since $\int_{\Omega} g_{2}(U) d x=0$, there exists $x_{1} \in \Omega$, such that $g_{2}\left(U\left(x_{1}\right)\right)=0$; that is,

$$
\sigma u^{n}\left(x_{1}\right)=b a+b u^{n}\left(x_{1}\right) .
$$

It follows that $\max _{\bar{\Omega}} u \geq \sqrt[n]{\mathrm{a} b / \sigma}$. Consequently, by $\max _{\bar{\Omega}} u / \min _{\bar{\Omega}} u \leq C$ in Theorem 1, we have

$$
\min _{\bar{\Omega}} u \geq \frac{1}{C} \max _{\bar{\Omega}} u \geq \frac{\sqrt[n]{a b / \sigma}}{C}:=C_{3}=C_{3}(d, \widehat{d}, P, \Omega),
$$

where $d_{3} \leq \widehat{d}$ and $d_{1}, d_{2} \geq d$.

In the following, we mainly prove that $\min _{\bar{\Omega}} v(x)>\underline{C}$ as $\sigma k^{n} \neq b\left(a+k^{n}\right)$. Now, we suppose that claim is not true; then, there exists a sequence $\left(d_{1, i}, d_{2, i}, d_{3, i}\right) \rightarrow\left(d_{1}, d_{2}, d_{3}\right)$ with $d_{1, i}, d_{2, i} \geq d$ and $d_{3, i} \leq \widehat{d}$. And the positive solution $\left(u_{i}, v_{i}\right)$ of $(1)$ corresponding to $\left(d_{1, i}, d_{2, i}, d_{3, i}\right)$ is such that

$$
u_{i} \longrightarrow u, \quad \min _{\bar{\Omega}} v_{i}(x) \longrightarrow 0, \quad \text { as } i \longrightarrow \infty .
$$

By (14), we have $\max _{\bar{\Omega}} v_{i}(x) / \min _{\bar{\Omega}} v_{i}(x) \leq C_{1}(1+\widehat{d})$, and then, $\max _{\bar{\Omega}} v_{i}(x) \rightarrow 0$; furthermore, $v_{i}(x) \rightarrow 0$ uniformly holds as $i \rightarrow \infty$. Then, the first equation of (1) becomes the following:

$$
\begin{gathered}
-\Delta u_{i}=\frac{1}{d_{1, i}}\left(1-\frac{u_{i}}{k}-\frac{u_{i}^{n-1} v_{i}}{a+u_{i}^{n}}\right) u_{i} \quad \text { in } \Omega, \\
\frac{\partial u_{i}}{\partial v}=0 \quad \text { on } \partial \Omega .
\end{gathered}
$$

According to (7), there exists $C=C(d, \widehat{d}, P, \Omega)$ such that $\left\|u_{i}\right\|_{\infty} \leq C,\left\|\left(1-u_{i} / k\right)-\left(u_{i}^{n-1} v_{i} /\left(a+u_{i}^{n}\right)\right) / d_{1, i}\left(1+d_{3, i} v_{i}\right)\right\|_{\infty} \leq$ $C$, where $C$ is a positive constant which does not depend on $i$. For each $i$ given in problem (24), it follows from $L^{p}$ estimate that $\left\|u_{i}\right\|_{W^{2, p}(\Omega)} \leq C^{2}|\Omega|^{1 / p}$, where $p>1$. Let $p>N$; then, by Sobolev Imbedding Theorems, we get

$$
\left\|u_{i}\right\|_{C^{1, \alpha}(\Omega)} \leq C^{*}\left\|u_{i}\right\|_{W^{2, p}(\Omega)} \leq C^{*} C^{2}|\Omega|^{1 / p},
$$

where $\alpha \in(0,1)$. We choose $\alpha<1-N / p$ such that Imbedding is compact, and along with elliptic equation regularity theory, there exists the subsequence of $\left\{u_{i}\right\}$, which is still denoted by $\left\{u_{i}\right\}$, and exists $u$ such that $u_{i} \rightarrow u$ uniformly holds in $C^{2, \alpha}(\Omega)$. On the other hand, $v_{i} \rightarrow 0$, and when $d_{1, i} \rightarrow d_{1} \epsilon$ $[d, \infty)$, the limit of (24) becomes the following problem:

$$
-\Delta u=\frac{1}{d_{1}} u\left(1-\frac{u}{k}\right) \quad \text { in } \Omega, \quad \frac{\partial u}{\partial \nu}=0 \quad \text { on } \partial \Omega .
$$

Applying Maximum Principle to problem (26) and noting that $\min _{\bar{\Omega}} u_{i} \geq C_{3}$, we have $u=k$.

However, when $d_{1, i} \rightarrow \infty$, the limit of (24) becomes the following problem:

$$
-\Delta u=0 \quad \text { in } \Omega, \quad \frac{\partial u}{\partial \nu}=0 \quad \text { on } \partial \Omega,
$$

which implies that $u=\widehat{C}$ for some nonnegative constant $\widehat{C}$. Since $\int_{\Omega} u_{i}\left(1-u_{i} / k-u_{i}^{n-1} v_{i} /\left(a+u_{i}^{n}\right)\right) d x=0$, by letting $i \rightarrow \infty$ 
and noting that $\min _{\bar{\Omega}} u_{i} \geq C_{3}, v_{i} \rightarrow 0$, and $u_{i} \rightarrow \widehat{C}$, we also have $u=k$.

By a similar argument as that in (24), for the second equation of (1), we can prove that there exists a subsequence in $C^{2, \alpha}(\Omega)$, such that $v_{i}(x) / \max _{\bar{\Omega}} v_{i}(x) \rightarrow v_{0}$. Since $v_{i} / \max _{\bar{\Omega}} v_{i}(x) \geq \min _{\bar{\Omega}} v_{i}(x) / \max _{\bar{\Omega}} v_{i}(x) \geq 1 / C(1+\widehat{d})$, then $v_{0} \geq 1 / C(1+\widehat{d})$. Dividing the second equation of (1) by $\max _{\bar{\Omega}} v_{i}(x)$, and integrating over $\Omega$, we have

$$
\int_{\Omega} \frac{v_{i}}{\max _{\bar{\Omega}} v_{i}(x)}\left(\frac{\sigma u_{i}^{n}}{a+u_{i}^{n}}-b\right) d x=0 .
$$

Let $i \rightarrow \infty$, and note that $v_{i} / \max _{\bar{\Omega}} v_{i}(x) \rightarrow v_{0}$ and $u_{i} \rightarrow k$; then, $\sigma k^{n} /\left(a+k^{n}\right)=b$. This contradiction to the assumption completes the proof.

\section{A Result on Degree Theory}

In this section, we obtain nonexistence of nonconstant positive solutions to (1) as $d_{3}=0$. Meanwhile, by degree theory, a general result to establish the existence of nonconstant positive solutions to (1) in the next section is proved.

Denote $\mathbf{d}=\left(d_{1}, d_{2}, d_{3}\right)$ and $P=(\sigma, a, k, b)$. We will fix $P \in(0, \infty)^{4}$ and take $\mathbf{d} \in(0, \infty)^{2} \times[0, \infty)$ as bifurcation parameters, the dependence of $P$ will often be suppressed. Define

$$
\begin{gathered}
X=\left\{U \in\left[C^{2}(\bar{\Omega})\right]^{2} \mid \frac{\partial U}{\partial \nu}=0 \text { on } \partial \Omega\right\}, \\
X^{+}=\{(u, v) \in X \mid u>0, v>0 \text { on } \bar{\Omega}\}, \\
B(C)=\left\{(u, v) \in X \mid \frac{1}{C}<u, v<C \text { on } \bar{\Omega}\right\} .
\end{gathered}
$$

Since

$$
\Phi_{U}(U)=\left[\begin{array}{cc}
d_{1} & 0 \\
-\frac{d_{2} d_{3} v}{(1+u)^{2}} & d_{2}\left[1+\frac{d_{3}}{(1+u)}\right]
\end{array}\right]
$$

and $\operatorname{det} \Phi_{U}(U)$ is positive for all nonnegative $U, \Phi_{U}^{-1}$ exists. Hence, $U$ is a positive solution to (1) if and only if

$$
\begin{aligned}
F(\mathbf{d} ; U):= & U-(I-\Delta)^{-1} \\
& \times\left\{\Phi_{U}(U)^{-1}\left[G(U)+\nabla U \Phi_{U U} \nabla U\right]+U\right\} \\
= & 0 \quad \text { in } X^{+},
\end{aligned}
$$

where $(I-\Delta)^{-1}$ is the inverse of $I-\Delta$ in $X$. As $F(\mathbf{d} ; \cdot)$ is a compact perturbation of the identity operator $I$, the LeraySchauder $\operatorname{deg}(F(\mathbf{d} ; \cdot), 0, B)$ is well defined if $F(\mathbf{d} ; U) \neq 0$ for all $U \in \partial B$.

By Theorems 1 and 2, there exists a positive constant $C_{0}(\mathbf{d}, P)$ such that if $F(\mathbf{d} ; U)=0$ in $X^{+}$, then $U \in B\left(C_{0}\right)$. Note that $C_{0}(\mathbf{d}, P)$ can be taken as a continuous function for $\sigma \neq b a / k^{n}+b$. By the invariance property of the LeraySchauder degree, we then conclude that $\operatorname{deg}(F(\mathbf{d} ; \cdot), 0, B(C))$ does not depend on $\mathbf{d}$ if $C>C_{0}(\mathbf{d}, P)$, and it also does not depend on $P$, if $P$ changes continuously in $(0, \infty))^{4}$ without touching the surface $\sigma=b a / k^{n}+b$.

For the case $\sigma>b a / k^{n}+b$, by degree invariance, we need only consider a special $\mathbf{d}$; say $\mathbf{d}=(d, d, 0)$ with large $d$. For this we can use the following nonexistence result. To compare the existence regions of (1) with and without cross-diffusion, we give a nonexistence result stronger than what is needed here.

Theorem 3. Let $\left(d_{2}, P\right) \in(0, \infty)^{5}$ be given. Then, there exists a positive constant $\widehat{d}_{1}\left(d_{2}, P\right)$ such that when $\mathbf{d}=\left(d_{1}, d_{2}, 0\right)$ and $d_{1} \geq \widehat{d}_{1}\left(d_{2}, P\right)$, (1) does not have any nonconstant positive solution.

Proof. First, by (7), there exists a positive constant $C=$ $C\left(P, \widehat{d}_{2}\right)$ such that a classical solution to (1) satisfies $u(x)$, $v(x) \leq C$ provided that $d_{2} \geq \widehat{d}_{2}$. Now, we write $\bar{f}$ as average of $f$ over $\Omega$, where

$$
f(u, v)=u\left(1-\frac{u}{k}-\frac{u^{n-1} v}{a+u^{n}}\right), \quad g(u, v)=\frac{\sigma u^{n} v}{a+u^{n}}
$$

Multiplying the equation for $u$ of $(1)$ by $(u-\bar{u})$ and integrating over $\Omega$ by parts, we have

$$
\begin{aligned}
d_{1} \int_{\Omega} \nabla|u-\bar{u}|^{2} d x & =\int_{\Omega} f(u, v)(u-\bar{u}) d x \\
& =\int_{\Omega}\{f(u, v)-f(\bar{u}, \bar{v})\}(u-\bar{u}) d x \\
& =\int_{\Omega}\left\{f_{u}(\xi, \eta)(u-\bar{u})^{2}\right. \\
& \left.+f_{v}(\xi, \eta)(u-\bar{u})(v-\bar{v})\right\} d x \\
& \leq \int_{\Omega}\left\{\left(C_{1} u-\bar{u}\right)^{2}+\varepsilon(v-\bar{v})^{2}\right\} d x
\end{aligned}
$$

for some positive constant $C_{1}=C_{1}\left(P, \widehat{d}_{2}\right)$ and $\varepsilon=\varepsilon\left(P, \widehat{d}_{2}\right) \ll$ 1 , where $\xi$ and $\eta$ lie between $u$ and $\bar{u}, v$ and $\bar{v}$, respectively. Similarly, we have

$$
\begin{aligned}
d_{2} \int_{\Omega} \nabla|v-\bar{v}|^{2} d x= & \int_{\Omega}[-b v+g(u, v)](v-\bar{v}) d x \\
= & \int_{\Omega}[-b(v-\bar{v})+g(u, v)-g(\bar{u}, \bar{v})] \\
& \times(v-\bar{v}) d x \\
= & \int_{\Omega}\left\{-b(v-\bar{v})^{2}+g_{v}(\xi, \eta)(v-\bar{v})^{2}\right. \\
& \left.+g_{u}(\xi, \eta)(u-\bar{u})(v-\bar{v})\right\} d x \\
\leq & \int_{\Omega}\left\{(\sigma-b)(v-\bar{v})^{2}+\varepsilon_{1}(v-\bar{v})^{2}\right. \\
& \left.+C_{2}(u-\bar{u})^{2}\right\} d x
\end{aligned}
$$


for some positive constant $C_{2}=C_{2}\left(P, \widehat{d}_{2}\right)$ and $\varepsilon_{1}=$ $\varepsilon_{1}\left(P, \widehat{\mathrm{d}}_{2}\right) \ll 1$, where $\xi$ and $\eta$ are the same as in (33). Adding (33) and (34), we obtain

$$
\begin{aligned}
& \int_{\Omega}\left\{d_{1} \nabla|u-\bar{u}|^{2}+d_{2} \nabla|v-\bar{v}|^{2}\right\} d x \\
& \quad \leq \int_{\Omega}\left\{\left(C_{1}+C_{2}\right)(u-\bar{u})^{2}+\left(\sigma-b+\varepsilon+\varepsilon_{1}\right)(v-\bar{v})^{2}\right\} d x .
\end{aligned}
$$

It follows from the Poincaré Inequality that

$$
\begin{aligned}
& \mu_{1} \int_{\Omega}\left\{d_{1}(u-\bar{u})^{2}+d_{2}(v-\bar{v})^{2}\right\} d x \\
& \quad \leq \int_{\Omega}\left\{\left(C_{1}+C_{2}\right)(u-\bar{u})^{2}+\left(\sigma-b+\varepsilon+\varepsilon_{1}\right)(v-\bar{v})^{2}\right\} d x .
\end{aligned}
$$

Since we can choose $d_{2} \geq \widehat{d}_{2}$ such that $\mu_{1} d_{2}>\sigma-b$, we may also choose $\varepsilon$ and $\varepsilon_{1}$ sufficiently small such that $\mu_{1} d_{2}>$ $\sigma-b+\varepsilon+\varepsilon_{1}$. Consequently, by (36),

$$
\mu_{1} d_{1} \int_{\Omega}(u-\bar{u})^{2} d x \leq\left(C_{1}+C_{2}\right) \int_{\Omega}(u-\bar{u})^{2} d x,
$$

which implies that $u=\bar{u}=$ constant, and, hence, $v=\bar{v}=$ constant if $d_{1} \geq \widehat{d}_{1}\left(d_{2}, P\right):=\left(C_{1}\left(P, \widehat{d}_{2}\right)+C_{2}\left(P, \widehat{d}_{2}\right)\right) / \mu_{1}$. Thus, we complete the proof of the theorem.

In the following, we only calculate $\operatorname{deg}(F, 0, B)$ when all solutions to $F=0$ are positive constant solutions in $B(C)$.

Let $0=\mu_{1}<\mu_{2}<\mu_{3}<\cdots$ be the eigenvalues of the operator $-\Delta$ in $\Omega$ with zero flux boundary condition, $E\left(\mu_{i}\right)$ be the eigenspace corresponding to $\mu_{i}$ in $C^{1}(\bar{\Omega}), \phi_{i j}, j=$ $1,2,3, \ldots, \operatorname{dim} E\left(\mu_{i}\right)$ an orthonormal basis of $E\left(\mu_{i}\right)$, and $X_{i j}=$ $\left\{C \phi_{i j} \mid C \in R^{2}\right\}$. Then, $X=\oplus_{i=1}^{\infty} X_{i}$, where $X_{i}=\oplus_{j=1}^{\operatorname{dim} E\left(\mu_{i}\right)} X_{i j}$. This decomposed method is similar to that of [22].

Let $U^{*}$ be a positive root to $G(U)=0$. We can calculate

$$
\begin{aligned}
D_{U} F\left(\mathbf{d} ; U^{*}\right)= & I-(I-\Delta)^{-1} \\
& \times\left\{\Phi_{U}^{-1}\left(U^{*}\right) G_{U}\left(U^{*}\right)+I\right\} \quad \text { in } L(X, X),
\end{aligned}
$$

where $L(X, X)$ is a linear mapping from $X$ to itself.

Denote that $H\left(\mathbf{d}, U^{*} ; \mu\right):=\operatorname{det}\left[\mu I-\Phi_{U}\left(U^{*}\right)^{-1} G_{U}\left(U^{*}\right)\right]$.

Lemma 4. Let $U^{*}$ be a positive root to $G(\cdot)=0$, and assume that $H\left(\mathbf{d}, U^{*} ; \mu_{i}\right) \neq 0$ for all $i$. Then,

$$
\text { index }\left(F(\mathbf{d} ; \cdot), U^{*}\right)=(-1)^{r}, \quad r=\sum_{i \geq 1, H\left(\mathbf{d}, \mu_{i}\right)<0} 1 .
$$

Proof. If $D_{U} F$ is invertible, then the index of $F$ at $U^{*}$ is defined as index $\left(F(\mathbf{d} ; \cdot), U^{*}\right)=(-1)^{r}$, where $r$ is the number of eigenvalues of $D_{U} F$ with negative real parts. The $\operatorname{deg}(F(\mathbf{d} ; \cdot), 0, B)$ is then equal to summation of the indexes over all solutions to $F=0$ in $B$, provided that $F \neq 0$ on $\partial B$. Since for each integer $i \geq 1, X_{i}$ is invariant under $D_{U}\left(F\left(\mathbf{d} ; U^{*}\right)\right)$, and $\lambda$ is an eigenvalue of $D_{U} F$ on $X_{i}$ if and only if $\lambda$ is an eigenvalue of following matrix:

$$
\begin{aligned}
& I-\frac{1}{1+\mu_{i}}\left[\Phi_{U}\left(U^{*}\right)^{-1} G_{U}\left(U^{*}\right)+I\right] \\
& \quad=\frac{1}{1+\mu_{i}}\left[\mu_{i} I-\Phi_{U}\left(U^{*}\right)^{-1} G_{U}\left(U^{*}\right)\right] .
\end{aligned}
$$

Since $H\left(d, U^{*} ; \mu\right) \neq 0, D_{U} F\left(\mathbf{d} ; U^{*}\right)$ is invertible. Therefore, the number of eigenvalues with negative real parts of $D_{U} F\left(\mathbf{d} ; U^{*}\right)$ on $X_{i}$ is odd if and only if $H\left(\mathbf{d}, U^{*} ; \mu_{i}\right)<0$, and therefore

$$
\operatorname{index}\left(F(\mathbf{d} ; \cdot), U^{*}\right)=(-1)^{r}, \quad r=\sum_{i \geq 1, H\left(\mathbf{d}, \mu_{i}\right)<0} 1 .
$$

Theorem 5. Assume that $P=(\sigma, a, k, b) \in(0, \infty)^{4}$ with $\sigma k^{n} \neq b\left(a+k^{n}\right)$. Then, for every $\mathbf{d} \in(0, \infty)^{2} \times[0, \infty)$, there exists a constant $C_{0}(\mathbf{d}, P)$ such that for every $C>C_{0}(\mathbf{d}, P)$,

$$
\operatorname{deg}(F(\mathbf{d} ; \cdot), 0, B(C))= \begin{cases}0, & \text { if } \sigma<\frac{b a}{k^{n}}+b, \\ 1, & \text { if } \sigma<\frac{b a}{k^{n}}+b .\end{cases}
$$

Proof. Since (1) does not have any positive solution in $B(C)$, when $\sigma \leq b$, we then conclude that

$$
\begin{array}{r}
\operatorname{deg}(F(\mathbf{d} ; \cdot), 0, B(C))=0, \\
\forall P \in\left\{(\sigma, a, k, b) \in(0, \infty)^{4} \mid \sigma<\frac{b a}{k^{n}}+b\right\}, \\
C>C_{0}(\mathbf{d}, P) .
\end{array}
$$

Next, to complete the proof of Theorem 5 . We need only to calculate the degree for the case $\sigma>b a / k^{n}+b$. In this case, by Theorem 3, all positive solutions to $F=0$ are the unique positive constant solution to $G(\cdot)=0$, denoted by $\widetilde{U}$, when $\mathbf{d}=(d, d, 0), \Phi_{U}(\widetilde{U})=d I$ so that $H(\mathbf{d}, \widetilde{U} ; \mu)=$ $(1 / d) \operatorname{det}\left(d \mu I-G_{U}(\widetilde{U})\right), \operatorname{det}\left(G_{U}(\widetilde{U})\right)=n b^{2}(\sigma-b) \widetilde{v} / \sigma^{2} \widetilde{u}>0$. This implies that when $d$ is sufficiently large, $H\left(\mathbf{d}, \widetilde{U} ; \mu_{i}\right)>0$ for all $i=1,2,3, \ldots$, and thus $r=0$. It follows from Lemma 4 that $\operatorname{deg}(F(\mathbf{d} ; \cdot), 0, B(C))=1$. This completes the proof.

Remark 6. The change of degree when $\sigma$ passes the borderline $\sigma=b a / k^{n}+b$ is due to the appearance (disappearance) of a positive constant steady-state bifurcating from $U \equiv(k, 0)$.

\section{Existence of Nonconstant Positive Solutions}

In this section, we establish the existence of positive nonconstant solutions for (1). In particular, we show that for certain ranges of parameters where (1) does not have any positive nonconstant steady state, our model can still produce patterns. The idea is as follows. First we calculate the index of $F(\mathbf{d} ; \cdot)$ at positive constant steady states. Suppose that the 
sum of all these indices is not equal to the degree stated in Theorem 5. Then, $F(\mathbf{d} ; \cdot)=0$ in $B(C)$ for $C=C_{0}(\mathbf{d}, P)$ must have a nonconstant positive solution, which also solves (1).

In the following, we always assume that $\sigma>b$, and (1) has a unique positive constant solution $(\tilde{u}, \widetilde{v})$, when $\widetilde{U}=(\widetilde{u}, \widetilde{v})$ is a positive solution to $G(U)=0$. We can get the following results at $\widetilde{U}$ by simply calculating

$$
\begin{aligned}
G_{U}(\widetilde{U}) & =\left[\begin{array}{cc}
\frac{1}{\sigma k}\{(n(\sigma-b)-2 \sigma) \widetilde{u}-k[n(\sigma-b)-\sigma]\} & -\frac{b}{\sigma} \\
\frac{n b(\sigma-b) \widetilde{v}}{\sigma \widetilde{u}} & 0
\end{array}\right] \\
& =:\left[\begin{array}{cc}
g_{11} & -\frac{b}{\sigma} \\
g_{21} & 0
\end{array}\right], \\
\Phi_{U}(\widetilde{U}) & =\left[\begin{array}{cc}
d_{1} \\
-\frac{d_{2} d_{3} \widetilde{v}}{(1+\widetilde{u})^{2}} & d_{2}\left[1+\frac{d_{3}}{(1+\widetilde{u})}\right]
\end{array}\right] \\
& =:\left[\begin{array}{cc}
\phi_{11} & 0 \\
\phi_{21} & \phi_{22}
\end{array}\right], \\
H(\mathbf{d}, \widetilde{U} ; \mu) & \equiv \operatorname{det}\left(\mu I-\Phi_{U}^{-1}(\widetilde{U}) G_{U}(\widetilde{U})\right) \\
& =\mu^{2}+\mu \frac{\left|\phi_{21}\right| b / \sigma-\phi_{22} g_{11}}{\phi_{11} \phi_{22}}+\frac{g_{21} b / \sigma}{\phi_{11} \phi_{22}} .
\end{aligned}
$$

To calculate the roots of $H(\mathbf{d}, \widetilde{U} ; \cdot)=0$, we will restrict our attention to large $|\mathbf{d}|$. Note that

$$
\lim _{d_{i} \rightarrow \infty} H(\mathbf{d}, \widetilde{U} ; \mu)=\mu^{2}-\Lambda_{i} \mu, \quad \forall i=2,3,
$$

where

$$
\begin{aligned}
\Lambda_{2}= & \Lambda_{2}\left(d_{1}, d_{3} ; U\right)=\left(-d_{3} b k \widetilde{v}+\left(1+\widetilde{u}+d_{3}\right)(1+\tilde{u})\right. \\
& \times\{(n(\sigma-b)-2 \sigma) \tilde{u}-k(n(\sigma-b)-\sigma)\}) \\
& \times\left(\sigma k d_{1}\left(1+\widetilde{u}+d_{3}\right)(1+\widetilde{u})\right)^{-1}, \\
\Lambda_{3}= & \Lambda_{3}\left(d_{1} ; U\right) \\
= & \frac{-b k \widetilde{v}+\{(n(\sigma-b)-2 \sigma) \tilde{u}-k(n(\sigma-b)-\sigma)\}(1+\tilde{u})}{\sigma k d_{1}(1+\tilde{u})} .
\end{aligned}
$$

The sign of the trace $\operatorname{tr}\left(G_{U}\right)=g_{11}$ is determined by $\gamma=$ $(n(\sigma-b)-2 \sigma) \tilde{u}-k(n(\sigma-b)-\sigma)$ and the sign of the determinant $\operatorname{det}\left(G_{U}\right)=n b^{2}(\sigma-b) v / \sigma^{2} u>0$.

Hence, we will discuss separately the following cases:

(i) $n(\sigma-b)-2 \sigma>0(n>2 \sigma /(\sigma-b))$, obviously $k>\tilde{u}>$ $((n(\sigma-b)-2 \sigma) /(n(\sigma-b)-\sigma)) \widetilde{u}$, then $\gamma<0 ;$

(ii) $n(\sigma-b)-\sigma<0(n<\sigma /(\sigma-b))$ :

(iia) if $\tilde{u}<k<((2 \sigma-n(\sigma-b)) /(\sigma-n(\sigma-b))) \tilde{u}$, then $\gamma<0$;

(iib) if $k>((2 \sigma-n(\sigma-b)) /(\sigma-n(\sigma-b))) \tilde{u}$, then $\gamma>0$.
4.1. The Case $n(\sigma-b)-2 \sigma>0(n>2 \sigma /(\sigma-b))$. In this subsection, we consider local stability of the constant steady state $U \equiv \widetilde{U}$ for evolution dynamics

$$
\begin{gathered}
U_{t}=\Delta \Phi(U)+G(U) \quad \text { in } \Omega \times(0, \infty), \\
\frac{\partial U}{\partial \nu}=0 \quad \text { on } \partial \Omega,
\end{gathered}
$$

where $\widetilde{U}=(\widetilde{u}, \widetilde{v})$ is a positive constant solution to $G(\cdot)=0$ by (5).

Theorem 7. Let $k>\widetilde{u}>((n(\sigma-b)-2 \sigma) /(n(\sigma-b)-$ $\sigma)) \tilde{u}(n>2 \sigma /(\sigma-b))$. Then the positive constant solution $U(x, t) \equiv(\widetilde{u}, \widetilde{v})$ is asymptotically stable with respect to the dynamics (47). Consequently, in a small neighborhood of $\widetilde{U}$, (1) does not have any nonconstant positive solution.

Proof. The linearization of (47) at $\widetilde{U}$ takes the form

$$
\begin{gathered}
V_{t}=\Phi_{U}(\widetilde{U}) \Delta V+G_{U}(\widetilde{U}) V \quad \text { in } \Omega \times(0, \infty), \\
\frac{\partial V}{\partial \nu}=0 \quad \text { on } \partial \Omega .
\end{gathered}
$$

Denote that the corresponding linear operator $L:=\Phi_{U}(\widetilde{U}) \Delta+$ $G_{U}(\widetilde{U})$. For each eigen-pair $\left(\mu_{i}, \phi_{i}\right)(i=1,2,3, \ldots)$ of $-\Delta$ on $\Omega$ with no flux boundary condition, $V=C e^{\lambda t} \phi$ is a solution to (48) if and only if $(\lambda, C)$ is an eigen-pair of the matrix $A_{i}:=$ $-\mu_{i} \Phi_{U}(\widetilde{U})+G_{U}(\widetilde{U}) . X_{i}$ is invariant under the operator $L$. From (44), we have

$$
g_{11}<0 \quad\left(k>\frac{n(\sigma-b)-2 \sigma}{n(\sigma-b)-\sigma} \tilde{u}\right), \quad g_{21}>0 \quad(\sigma>b) .
$$

Then

$$
\begin{aligned}
\operatorname{det} A_{i}:= & \phi_{11} \phi_{22} \mu_{i}^{2}-\left(\frac{b}{\sigma} \phi_{21}+g_{11} \phi_{22}\right) \mu_{i}+\frac{b}{\sigma} g_{21}>0 \\
& \operatorname{tr} A_{i}:=-\left(\phi_{11}+\phi_{22}\right) \mu_{i}+g_{11}<0
\end{aligned}
$$

Hence, we conclude that the two eigenvalues $\lambda_{i}^{+}$and $\lambda_{i}^{-}$of $A_{i}$ have negative real parts. More specifically, we have the following:

(i) For $i=1, \mu_{1}=0$, if $g_{11}^{2}-4 g_{21} b / \sigma \leq 0$, then $\operatorname{Re} \lambda_{i}^{ \pm}=$ $(1 / 2) g_{11}<0$; if $g_{11}^{2}-4 g_{21} b / \sigma>0$, then

$$
\begin{aligned}
& \operatorname{Re} \lambda_{i}^{+}=\frac{1}{2}\left[g_{11}+\sqrt{g_{11}^{2}-\frac{4 g_{21} b}{\sigma}}\right] \leq 0, \\
& \operatorname{Re} \lambda_{i}^{-}=\frac{1}{2}\left[g_{11}-\sqrt{g_{11}^{2}-\frac{4 g_{21} b}{\sigma}}\right]<0 .
\end{aligned}
$$


(ii) For $i \geq 2$, as $\mu_{i}$ is increasing with respect to $i$ and $\mu_{i} \rightarrow$ $\infty$ as $i \rightarrow \infty$, it follows that if $\left(\operatorname{tr} A_{i}\right)^{2}-4 \operatorname{det} A_{i} \leq 0$, then

$$
\begin{aligned}
\operatorname{Re} \lambda_{i}^{ \pm} & =\frac{1}{2} \operatorname{tr} A_{i}=\frac{1}{2}\left[-\left(\phi_{11}+\phi_{22}\right) \mu_{i}+g_{11}\right] \\
& \leq \frac{1}{2}\left[-\left(\phi_{11}+\phi_{22}\right) \mu_{2}+g_{11}\right]<0 ;
\end{aligned}
$$

if $\left(\operatorname{tr} A_{i}\right)^{2}-4 \operatorname{det} A_{i}>0$, since $\operatorname{det} A_{i}>0$ and $\operatorname{tr} A_{i}<0$, then

$$
\begin{aligned}
\operatorname{Re} \lambda_{i}^{-} & =\frac{1}{2}\left(\operatorname{tr} A_{i}-\sqrt{\left(\operatorname{tr} A_{i}\right)^{2}-4 \operatorname{det} A_{i}}\right) \\
& \leq \frac{1}{2} \operatorname{tr} A_{i} \leq \frac{1}{2}\left[-\left(\phi_{11}+\phi_{22}\right) \mu_{2}+g_{11}\right]<0 ; \\
\operatorname{Re} \lambda_{i}^{+} & =\frac{1}{2}\left(\operatorname{tr} A_{i}+\sqrt{\left(\operatorname{tr} A_{i}\right)^{2}-4 \operatorname{det} A_{i}}\right) \\
& =\frac{2 \operatorname{det} A_{i}}{\operatorname{tr} A_{i}-\sqrt{\left(\operatorname{tr} A_{i}\right)^{2}-4 \operatorname{det} A_{i}}} \leq \frac{\operatorname{det} A_{i}}{\operatorname{tr} A_{i}}<-\widetilde{\varepsilon} .
\end{aligned}
$$

for some positive constant $\widetilde{\varepsilon}$ that does not depend on $i$. The previous arguments show that there exists a positive constant $\varepsilon$, which does not depend on $i$, such that $\operatorname{Re} \lambda_{i}<$ $-\varepsilon, \forall i$. Consequently, the spectrum of $L$, which consists of eigenvalues, lies in $\{\operatorname{Re}(\lambda)<-\varepsilon\}$. It then follows from Theorem 5.1.1 of [26, page 98] that the constant steady-state $U(x, t) \equiv \widetilde{U}$ is asymptotically stable to (47).

\subsection{The Case $n(\sigma-b)-\sigma<0(n<\sigma /(\sigma-b))$}

4.2.1. $(\gamma<0) \tilde{u}<k<((2 \sigma-n(\sigma-b)) /(\sigma-n(\sigma-b))) \tilde{u}$

Theorem 8. Let $\tilde{u}<k<((2 \sigma-n(\sigma-b)) /(\sigma-n(\sigma-b))) \tilde{u}$. Then the positive constant solution $U(x, t) \equiv(\widetilde{u}, \widetilde{v})$ is asymptotically stable with respect to the dynamics (47).

Proof. Similar to the proof of Theorem 7.

4.2.2. $(\gamma>0) k>((2 \sigma-n(\sigma-b)) /(\sigma-n(\sigma-b))) \tilde{u}$. In this case, $\widetilde{U}=(\widetilde{u}, \widetilde{v})$ is the only positive constant solution to $G(\cdot)=0$. By fixing the diffusion coefficients $d_{1}$ (for prey) and using the diffusion coefficients $d_{2}$ and $d_{3}$ (for predator) as bifurcation parameters, we will show that (1) can create nonconstant positive solutions. We want to emphasize that it is caused by the presence of cross-diffusion which has a more complex role than that of the diffusion coefficients $d_{1}$ and $d_{2}$.

Theorem 9 (existence with suitable $d_{2}$ and $d_{3}$ ). Assume that $\sigma>b a / k^{n}+b$ and $k>((2 \sigma-n(\sigma-b)) /(\sigma-n(\sigma-b))) \tilde{u}$, define $\Lambda_{2}\left(d_{1}, d_{3} ; U\right)$ and $\Lambda_{3}\left(d_{1} ; U\right)$ as $(46)$.

(i) Suppose that $d_{1}$ and $d_{3}$ are given such that $\Lambda_{2}\left(d_{1}, d_{3} ; \widetilde{U}\right) \in\left(\mu_{j}, \mu_{j+1}\right)$ for some positive even integer $j$. There exists a positive constant $D_{2}$ such that if $d_{2} \geq D_{2}$, then

(1) has at least one nonconstant positive solution.

(ii) Suppose that $d_{1}$ is given such that $\Lambda_{3}\left(d_{1} ; U\right) \in$ $\left(\mu_{j}, \mu_{j+1}\right)$ for some positive even integer $j$. Then, for any given $d_{2}>0$, there exists a positive constant $D_{3}$ such that if $d_{3} \geq D_{3}$, (1) has at least one nonconstant positive solution.
Proof. Denote by $\mu^{ \pm}(\mathbf{d}, \widetilde{U})$, with $\operatorname{Re}\left(\mu^{-}\right) \leq \operatorname{Re}\left(\mu^{+}\right)$, the two roots to $H(\mathbf{d}, \widetilde{U} ; \mu)=0$, and then

$$
\begin{aligned}
\mu^{+}(\mathbf{d}, \widetilde{U}) \mu^{-}(\mathbf{d}, \widetilde{U}) & =\left[\operatorname{det}\left(\Phi_{U}(\widetilde{U})\right)\right]^{-1} \operatorname{det}\left(G_{U}(\widetilde{U})\right) \\
& =\frac{g_{21} b / \sigma}{\phi_{11} \phi_{22}}>0 .
\end{aligned}
$$

From (45), we see that

$$
\begin{array}{lc}
\lim _{d_{2} \rightarrow \infty} \mu^{-}(\mathbf{d}, \widetilde{U})=0, & \lim _{d_{2} \rightarrow \infty} \mu^{+}(\mathbf{d}, \widetilde{U})=\Lambda_{2}\left(d_{1}, d_{3} ; \widetilde{U}\right), \\
\lim _{d_{3} \rightarrow \infty} \mu^{-}(\mathbf{d}, \widetilde{U})=0, & \lim _{d_{3} \rightarrow \infty} \mu^{+}(\mathbf{d}, \widetilde{U})=\Lambda_{3}\left(d_{1} ; \widetilde{U}\right) .
\end{array}
$$

Suppose that $\Lambda_{2}\left(d_{1}, d_{3} ; \widetilde{U}\right) \in\left(\mu_{j}, \mu_{j+1}\right)$ for some positive even integer $j$; then, there exists a positive constant $D_{2} \gg 1$ such that $d_{2} \gg D_{2}$, and we have

$$
0=\mu_{1}<\mu^{-}(\mathbf{d}, \widetilde{U})<\mu_{2}, \quad \mu^{+}(\mathbf{d}, \widetilde{U}) \in\left(\mu_{j}, \mu_{j+1}\right) .
$$

Hence, if $d_{2} \gg 1, H\left(\mathbf{d}, \widetilde{U} ; \mu_{i}\right)<0$ is equivalent to $i \in$ $\{2,3, \ldots, j\}$, since $j$ is even. It follows from Lemma 4 that

$$
\operatorname{index}(F(\mathbf{d} ; \cdot), \widetilde{U})=(-1)^{j-1}=-1 .
$$

Consequently, $F(\mathbf{d} ; U)=0$ has at least one nonconstant positive solution that is different from the constant function $U=\widetilde{U}$. Otherwise, the degree of $F=0$ in $B(C)$ would be -1 for all large enough $C$, which would contradict Theorem 5 . This proves, the first assertion of the theorem, and the second assertion is similarly proved.

Remark 10. For $\Lambda_{3}\left(d_{1} ; U\right)$ to be positive, it is necessary and sufficient to have

$$
k>\frac{(k+1) \sigma \tilde{u}}{(1+\tilde{u})[\sigma-n(\sigma-b)]}+\tilde{u} .
$$

When this inequality holds, $\Lambda_{2}\left(d_{1}, d_{3} ; \widetilde{U}\right)$ is also positive provided that $d_{3}$ is large, and we then can adjust to $d_{1}$ and make the assumptions in (i) or (ii) of theorem hold.

Remark 11. In fact, if $\sigma /(\sigma-b)<n<2 \sigma /(\sigma-b)$, then $\gamma<$ 0 . This case is similar to case (i). Furthermore, the positive constant solution $\mathrm{U}(x, t) \equiv(\widetilde{u}, \widetilde{v})$ is also asymptotically stable with respect to the dynamics (47).

\section{Acknowledgments}

This project is supported by the NSF of China under Grant 11001160, the Scientific Research Plan Projects of Shaanxi Education Department (no. 12JK0865), and the President Fund of Xi'an Technological University (XAGDXJJ1136).

\section{References}

[1] W. Wang and J. H. Sun, "On the predator-prey system with Holling- $(n+1)$ functional response," Acta Mathematica Sinica, vol. 23, no. 1, pp. 1-6, 2007. 
[2] J. A. Johnson, "Banach spaces of Lipschitz functions and vectorvalued Lipschitz functions," Transactions of the American Mathematical Society, vol. 148, pp. 147-169, 1970.

[3] X. F. Chen, Y. W. Qi, and M. X. Wang, "A strongly coupled predator-prey system with non-monotonic functional response," Nonlinear Analysis. Theory, Methods \& Applications, vol. 67, no. 6, pp. 1966-1979, 2007.

[4] T. Kadota and K. Kuto, "Positive steady states for a preypredator model with some nonlinear diffusion terms," Journal of Mathematical Analysis and Applications, vol. 323, no. 2, pp. 1387-1401, 2006.

[5] N. Shigesada, K. Kawasaki, and E. Teramoto, "Spatial segregation of interacting species," Journal of Theoretical Biology, vol. 79, no. 1, pp. 83-99, 1979.

[6] H. Amann, "Dynamic theory of quasilinear parabolic systems. III. Global existence," Mathematische Zeitschrift, vol. 202, no. 2, pp. 219-250, 1989.

[7] Y. S. Choi, R. Lui, and Y. Yamada, "Existence of global solutions for the Shigesada-Kawasaki-Teramoto model with strongly coupled cross-diffusion," Discrete and Continuous Dynamical Systems A, vol. 10, no. 3, pp. 719-730, 2004.

[8] L. Dung, "Cross diffusion systems on $n$ spatial dimensional domains," Indiana University Mathematics Journal, vol. 51, no. 3, pp. 625-643, 2002.

[9] L. Dung, L. V. Nguyen, and T. T. Nguyen, "Shigesada-KawasakiTeramoto model on higher dimensional domains," Electronic Journal of Differential Equations, vol. 2003, pp. 1-12, 2003.

[10] Y. Lou, W.-M. Ni, and Y. Wu, "On the global existence of a crossdiffusion system," Discrete and Continuous Dynamical Systems, vol. 4, no. 2, pp. 193-203, 1998.

[11] A. Yagi, "Global solution to some quasilinear parabolic system in population dynamics," Nonlinear Analysis. Theory, Methods \& Applications, vol. 21, no. 8, pp. 603-630, 1993.

[12] Y. Kan-on, "Stability of singularly perturbed solutions to nonlinear diffusion systems arising in population dynamics," Hiroshima Mathematical Journal, vol. 23, no. 3, pp. 509-536, 1993.

[13] K. Kuto, "Stability of steady-state solutions to a prey-predator system with cross-diffusion," Journal of Differential Equations, vol. 197, no. 2, pp. 293-314, 2004.

[14] Y. Wu, "The instability of spiky steady states for a competing species model with cross diffusion," Journal of Differential Equations, vol. 213, no. 2, pp. 289-340, 2005.

[15] K. Kuto and Y. Yamada, "Multiple coexistence states for a preypredator system with cross-diffusion," Journal of Differential Equations, vol. 197, no. 2, pp. 315-348, 2004.

[16] Y. Lou and W.-M. Ni, "Diffusion vs cross-diffusion: an elliptic approach," Journal of Differential Equations, vol. 154, no. 1, pp. 157-190, 1999.

[17] Y. Lou, W.-M. Ni, and S. Yotsutani, "On a limiting system in the Lotka-Volterra competition with cross-diffusion," Discrete and Continuous Dynamical Systems A, vol. 10, no. 1-2, pp. 435-458, 2004.

[18] M. Mimura, Y. Nishiura, A. Tesei, and T. Tsujikawa, "Coexistence problem for two competing species models with densitydependent diffusion," Hiroshima Mathematical Journal, vol. 14, no. 2, pp. 425-449, 1984.

[19] K. Nakashima and Y. Yamada, "Positive steady states for preypredator models with cross-diffusion," Advances in Differential Equations, vol. 1, no. 6, pp. 1099-1122, 1996.
[20] W. H. Ruan, "Positive steady-state solutions of a competing reaction-diffusion system with large cross-diffusion coefficients," Journal of Mathematical Analysis and Applications, vol. 197, no. 2, pp. 558-578, 1996.

[21] K. Ryu and I. Ahn, "Positive steady-states for two interacting species models with linear self-cross diffusions," Discrete and Continuous Dynamical Systems A, vol. 9, no. 4, pp. 1049-1061, 2003.

[22] P. Y. H. Pang and M. X. Wang, "Strategy and stationary pattern in a three-species predator-prey model," Journal of Differential Equations, vol. 200, no. 2, pp. 245-273, 2004.

[23] P. Y. H. Pang and M. Wang, "Qualitative analysis of a ratiodependent predator-prey system with diffusion," Proceedings of the Royal Society of Edinburgh A, vol. 133, no. 4, pp. 919-942, 2003.

[24] C.-S. Lin, W.-M. Ni, and I. Takagi, "Large amplitude stationary solutions to a chemotaxis system," Journal of Differential Equations, vol. 72, no. 1, pp. 1-27, 1988.

[25] Y. Lou and W.-M. Ni, "Diffusion, self-diffusion and crossdiffusion," Journal of Differential Equations, vol. 131, no. 1, pp. 79-131, 1996.

[26] D. Henry, Geometric Theory of Semilinear Parabolic Equations, vol. 840 of Lecture Notes in Mathematics, Springer, Berlin, Germany, 3rd edition, 1981. 


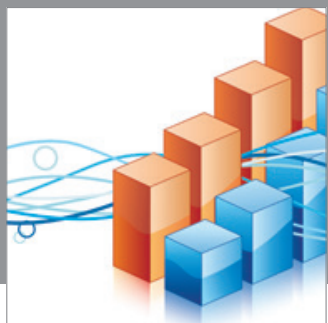

Advances in

Operations Research

mansans

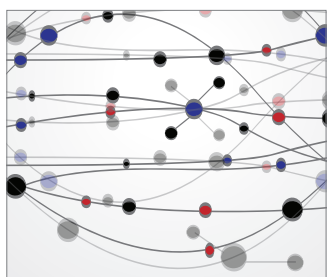

The Scientific World Journal
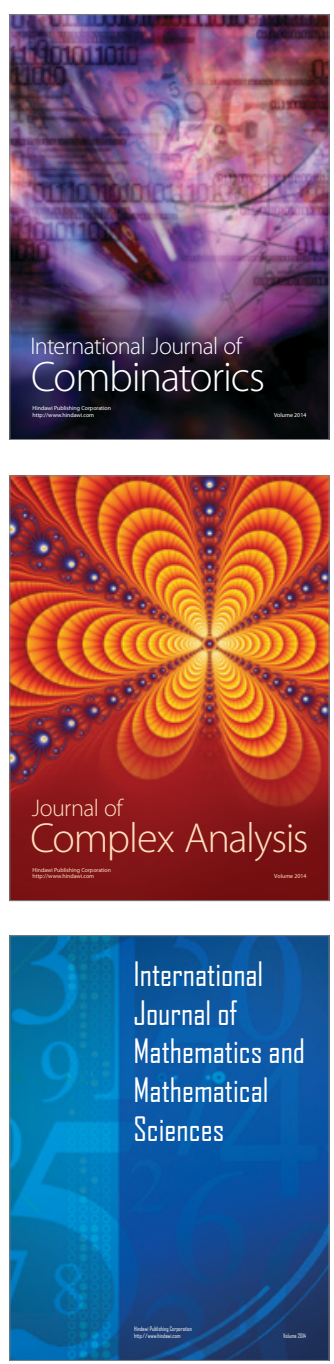
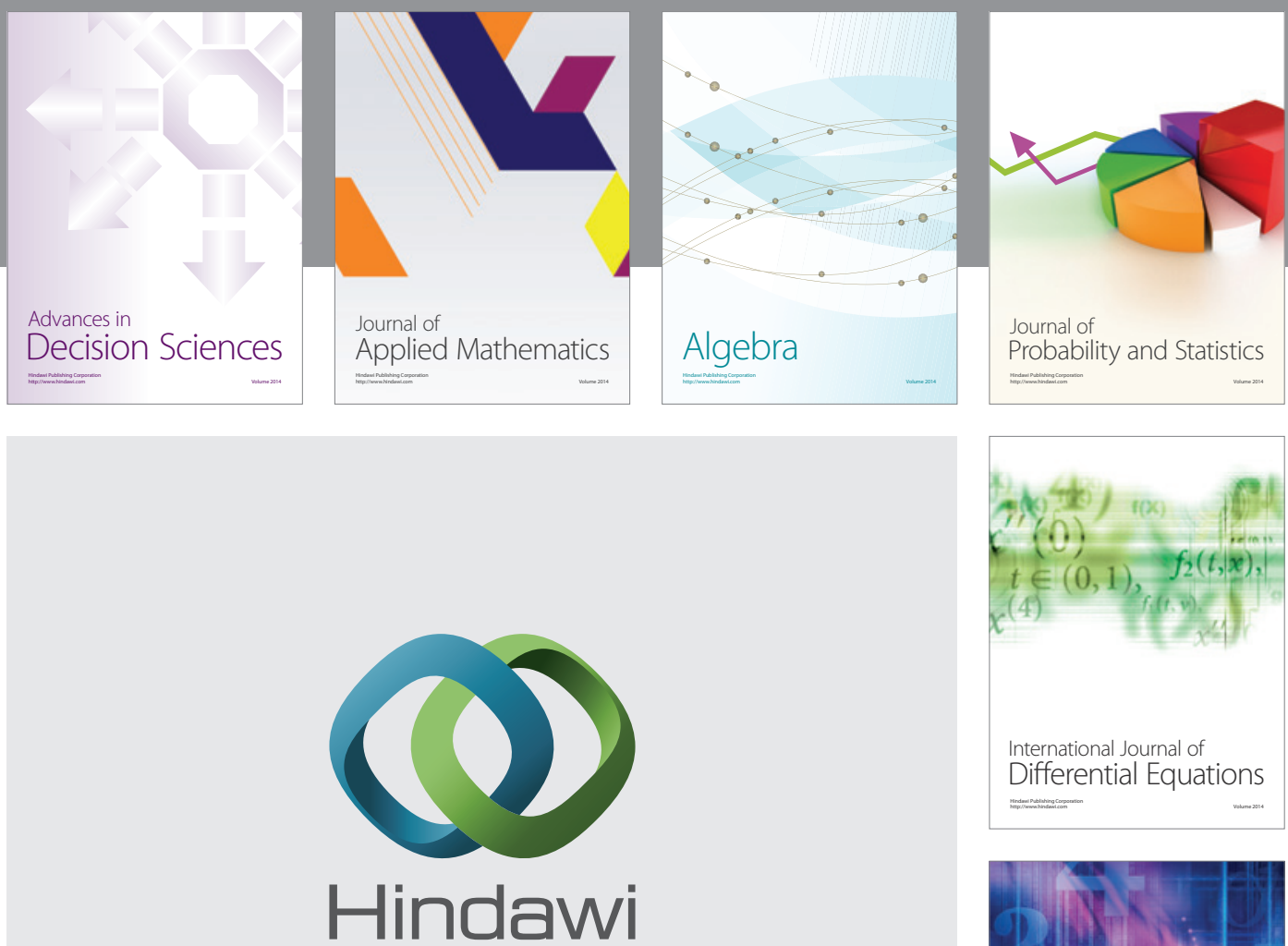

Submit your manuscripts at http://www.hindawi.com
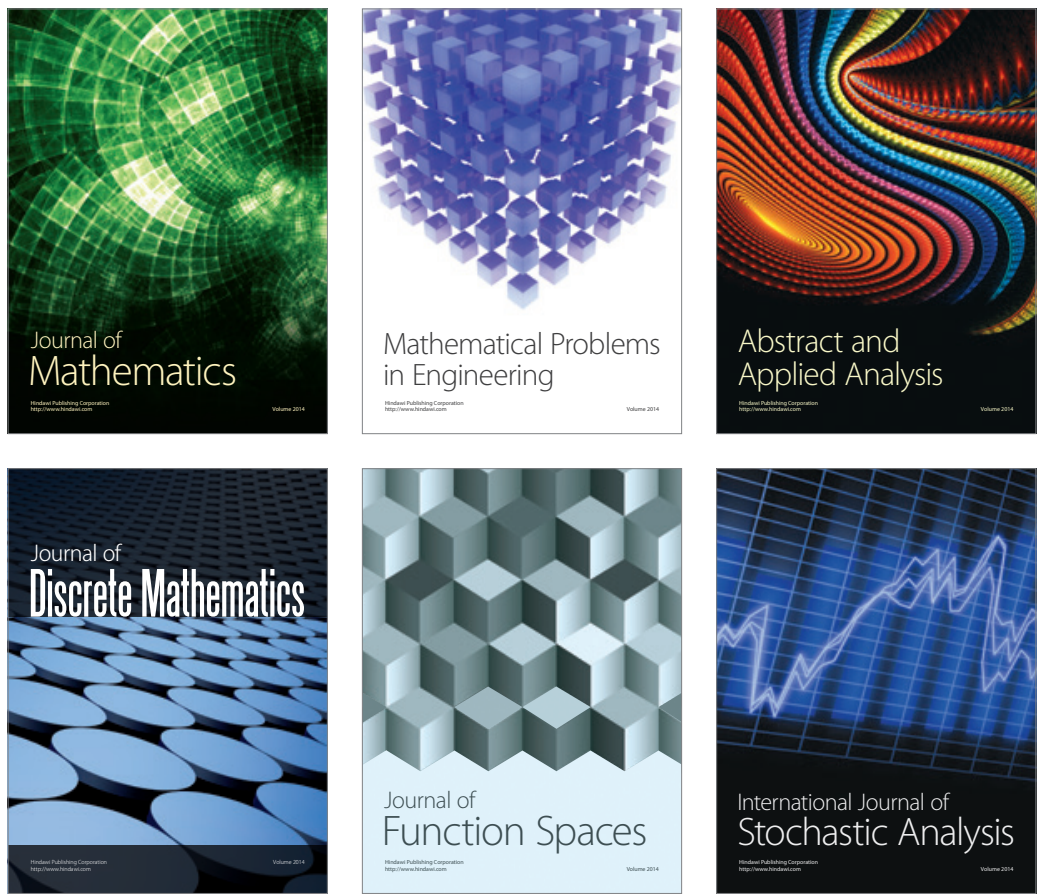

Journal of

Function Spaces

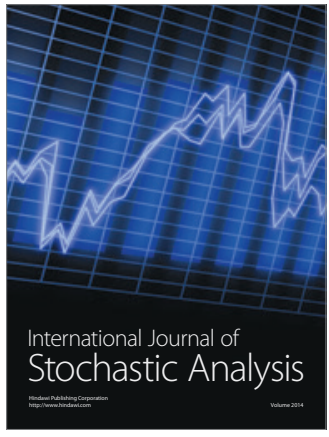

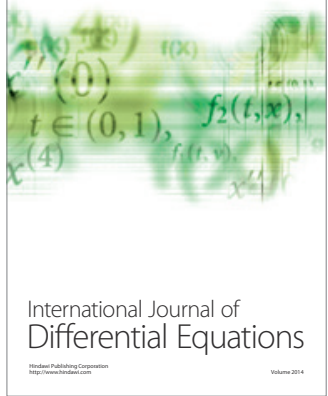
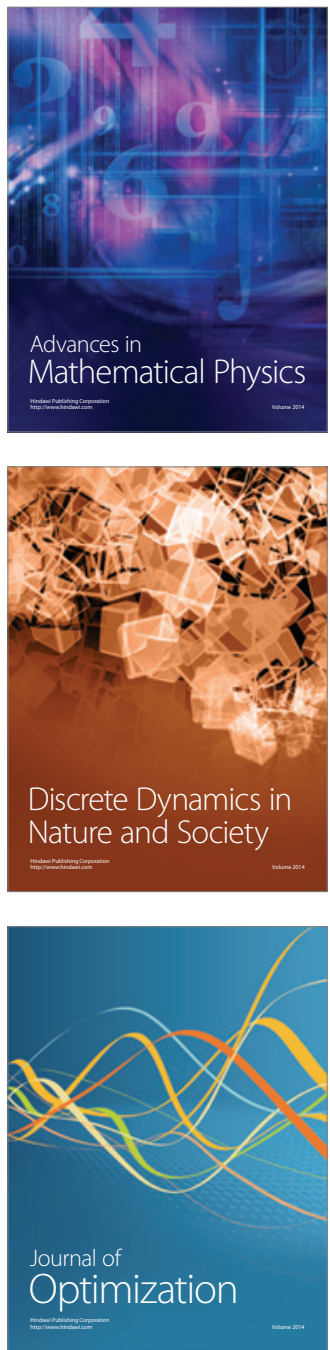